\title{
PRÁCTICAS SUSTENTABLES DE MEJORAMIENTO DE SUELO EN SISTEMAS HORTÍCOLAS EN EL MARCO DE LA EXTENSIÓN UNIVERSITARIA
}

\author{
Soil improvement sustainable practices in horticultural systems by the universitary \\ extension \\ Carnicer, Sebastián; Sotelo, Cristina E.; Pérez, Germán L., Ballatore, Bárbara M.; Castelán, \\ María E. \\ Instituto Agrotécnico "Pedro M. Fuentes Godo" FCA - Universidad Nacional del Nordeste \\ Av. Las Heras 727 - Resistencia - Chaco - Argentina \\ E-mail: institutoagrotécnicounne@hotmail.com
}

\begin{abstract}
RESUMEN
Las Buenas Prácticas Agrícolas han sido incorporadas al Código Alimentario Argentino, según lo resuelto a partir del 2021 entran en vigencia para el sector hortícola. Debido a esto es indispensable acompañar los procesos productivos, entendiendo que el aprendizaje se basa en la propia experiencia y es fruto de interacciones colectivas. El objetivo de este trabajo fue promover la adopción de tecnologías para mejorar la fertilidad de suelo, como la incorporación de abono verde y compost en sistemas hortícolas, a través de talleres y experiencias demostrativas. Además, facilitar la interacción de los estudiantes, productores y técnicos de las distintas instituciones. Las experiencias se dividieron en etapas: identificación del problema; desarrollo de una conciencia crítica; propuesta; seguimiento de la actividad; evaluación, discusión y reflexión; jornada de campo con productores demostrativos y aporte de material técnico. Se logró un trabajo en equipo entre los distintos actores, estudiantes, productores y profesionales de las instituciones involucradas. Los alumnos aplicaron los conocimientos teóricos adquiridos, al llevarlos a la práctica, en tareas como muestreo de suelo y análisis de suelo y compost. También conocieron la realidad que afronta el productor en su vida laboral cotidiana e interactuar en los procesos de generación del conocimiento en la educación no formal. En cuanto a los productores, mostraron predisposición e interés por las prácticas recomendadas. También se afianzó el vínculo laboral y de respeto mutuo entre ellos y los profesionales de las distintas instituciones participantes, logrando un espacio coparticipativo y dinámico.
\end{abstract}

Palabras clave: compost, abonos verdes, horticultura.

\begin{abstract}
Good Agricultural Practices have been incorporated into the Argentine Food Code and it will come into effect within the horticultural system in 2021. Due to this, it is essential to go along with production processes, bearing in mind that learning is based on self experience and is the result of collective interactions. The aim of this work was to foster agricultural technologies so as to improve soil fertility through workshops and demonstration experiences in which students, producers and technicians from different institutions interact in the practices of green manure and compost. The experiences were divided into stages: problem identification; critical awareness development; proposal; activity monitoring; assessment; discussion and reflection; field day with farm producers with role models and technical material provision. Teamwork was carried out between the different participants in the projects: students, producers and professionals from the institutions involved. Students applied the acquired technical knowledge to perform different tasks: such as soil sampling and soil and compost analysis. They got to know what producers' working daily lifestyle was like and took part in the processes of generating knowledge in non-formal education contexts. Producers have shown deep interest in recommended practices. The labour entailment and mutual respect among all participants was also strengthened by achieving dynamic and partnership space.
\end{abstract}

Key words: compost, green manures, horticulture.

Recibido: 17/jun/2020. Aceptado: 08/jul/2020 


\section{INTRODUCCIÓN}

El suelo es considerado un sistema vivo, complejo y dinámico en equilibrio, pero que reacciona a determinadas acciones antrópicas o estímulos (Chilon Camacho, 2018). Con la llamada "Revolución Verde", los suelos fueron sometidos a distintos niveles de degradación por el uso de insumos sintéticos y mecanización (Ceccon, 2008; Pazos-Rojas et al., 2016). La Organización de las Naciones Unidas para la Alimentación y la Agricultura (FAO) señaló que una de las causas principales de la degradación de los suelos en América Latina es la aplicación de técnicas de labranzas inadecuadas. Esta práctica estimula la mineralización de la materia orgánica y en consecuencia afecta a las propiedades del suelo y del medio ambiente. La magnitud de su efecto depende, entre otras cosas, del grado de intensidad de las labranzas (Deagustini et al., 2017; Damian Suclupe et al., 2018). Además el uso de fertilizantes sintéticos (y aún más en altas dosis) a través de los años, también incide en la degradación y contaminación del suelo y el sistema agrícola en general (Martín et al., 2017; Marca-Huamancha et al., 2018). De allí la importancia del contenido de materia orgánica en los suelos, ya que le proporciona gran parte de la capacidad para almacenar nutrientes, agua y biodiversidad. También desempeña un papel fundamental en la formación y estabilización de la estructura del suelo, lo que a su vez promueve el drenaje y brinda una mejor resistencia a la erosión (Weil y Magdoff, 2004; Orozco Corral et al., 2016).

El sistema de producción hortícola regional se caracteriza por la extracción continua de nutrientes del suelo, la disminución del contenido de materia orgánica y la degradación de la estructura superficial. Debido a esto, pierden calidad y aptitud para funcionar afectando la sustentabilidad del agroecosistema por problemas de salinidad, alcalinidad, hiperfertilización y en muchos casos pérdida de materia orgánica (Sasal et al., 2009; Cuellas et al., 2018).

Por otro lado, las Buenas Prácticas Agrícolas han sido incorporadas al Código Alimentario Argentino (CAA) y según lo resuelto, a partir del año 2021 entrarán en vigencia para el sector hortícola (Resolución Conjunta 5/2018). Esto implica la necesidad de mantener acciones que promuevan la correcta aplicación de prácticas agrícolas con la finalidad, no solamente de cumplir con una resolución nacional, sino también de mitigar los efectos del manejo inapropiado que se realiza en la zona. Debido a esto es indispensable acompañar los procesos productivos, para que los productores puedan desarrollarse conforme a las necesidades y condiciones de los nuevos paradigmas. Reforzando la idea de que el aprendizaje se basa en la propia experiencia y es fruto de interacciones colectivas entre el hombre y su entorno, ya sean padres, hijos o vecinos (Valentinuz et al., 2005).

El Instituto Agrotécnico "Pedro M. Fuentes Godo" trabaja desde el año 1956 en proyectos de extensión rural y transferencia de tecnologías vinculadas a evitar la degradación de los suelos y en particular al manejo del carbono y el nitrógeno. Actualmente, a través de proyectos de extensión se continúa trabajando con este objetivo en el nuevo escenario que se plantea, tanto productivo como social. En estos proyectos participan estudiantes de distintas carreras, en busca de promover la utilidad social del conocimiento adquirido y la práctica solidaria y formativa. Dado que el aprendizaje sobre esta temática no es realizado dentro de un entorno académico formal, a través de estos trabajos se les brinda el medio físico y social para su desarrollo (Valentinuz et al., 2005; Bejarano, 2011).

El objetivo de este trabajo fue promover la adopción de tecnologías para mejorar la fertilidad de suelo, como la incorporación de abono verde y compost, en sistemas hortícolas del Cinturón Verde del Gran Resistencia. Además, facilitar la interacción de los estudiantes, productores y técnicos de las distintas instituciones participantes.

\section{METODOLOGÍA Y RESULTADOS OBTENIDOS}

El trabajo de los últimos seis años fue enmarcado en proyectos anuales del programa "la Universidad en el Medio". Este programa convoca a distintos actores de la comunidad universitaria, de instituciones y miembros de la comunidad en general a trabajar sobre alguna temática para mejorar la calidad de vida de la sociedad. El Instituto Agrotécnico, por medio de sus técnicos profesionales, docentes investigadores, no docentes y estudiantes universitarios, participó de proyectos orientados a la producción sustentable poniendo énfasis en el cuidado del suelo. 
Carnicer, S. et al.: Prácticas sustentables de mejoramiento de suelo.

Dichos proyectos tienen la característica de ser interinstitucionales, por lo que se trabajó con técnicos del Ministerio de Producción de la Provincia del Chaco, de la Secretaría de Agricultura Familiar, del Instituto Nacional de Tecnología Agropecuaria (INTA) y de Cooperativas de productores.

Inicialmente se realizaron reuniones con profesionales de las instituciones mencionadas y se coordinaron visitas a productores hortícolas de distintas escalas.

En cada visita a los establecimientos hortícolas, se enfatizó la importancia de implementar las buenas prácticas para mejorar o conservar el suelo. De acuerdo a las posibilidades de cada productor y grupo de profesionales, se decidió qué prácticas se implementarían en cada establecimiento. Los establecimientos, donde se realizarían las experiencias, fueron considerados como "productores demostradores". En cada caso se planificaron y se proyectaron las actividades junto a productores, técnicos y alumnos.

Dado el gran laboreo de suelo que se realiza en la actividad hortícola se planteó la necesidad de mantener o mejorar la fertilidad química y aumentar el contenido de materia orgánica del suelo. Se decidió abordar el tema desde la realización de ensayos de abonos verdes, promoción del compost, y talleres demostrativos.

Las experiencias, se dividieron en etapas:

1. Identificación y desarrollo de una conciencia crítica del problema: en los establecimientos seleccionados, se realizaron los análisis químicos necesarios para diagnosticar el problema y marcar un punto de partida para las experiencias demostrativas.

2. Propuesta para solucionar el problema: posteriormente se analizaron los resultados encontrados y se realizó la devolución de los mismos a cada productor, explicando las alternativas de prácticas sustentables para mejorar el suelo.

3. Seguimiento de la actividad: Periódicamente, se realizaron visitas a los productores para acompañar el desarrollo de la experiencia logrando la integración, participación y capacitación de los mismos. Además de esto, se logró un grado de confianza que permitió que los productores planteen dudas y propuestas sobre la temática, resultando en un intercambio de conocimientos y comunicación enriquecedor.

4. Talleres de campo con productores demostradores.

5. Aporte de material técnico (folletos) sobre la experiencia.

\section{Experiencias con abonos verdes}

Los abonos verdes, cultivos que tienen como finalidad primaria su incorporación al suelo, siendo su principal condición que se encuentren en estado vegetativo o en floración para mejorar su aporte en fertilidad edáfica (Martín y Rivera, 2004; Castro-Rincón et al., 2018). Para trabajar con esta temática se buscaron productores interesados, a quienes se les explicaron los beneficios de la práctica y se determinó en cada caso el lote donde se realizarían. Para esta experiencia se utilizó una combinación de una leguminosa (Vicia villosa) y una gramínea (Avena strigosa) (Figura 1).

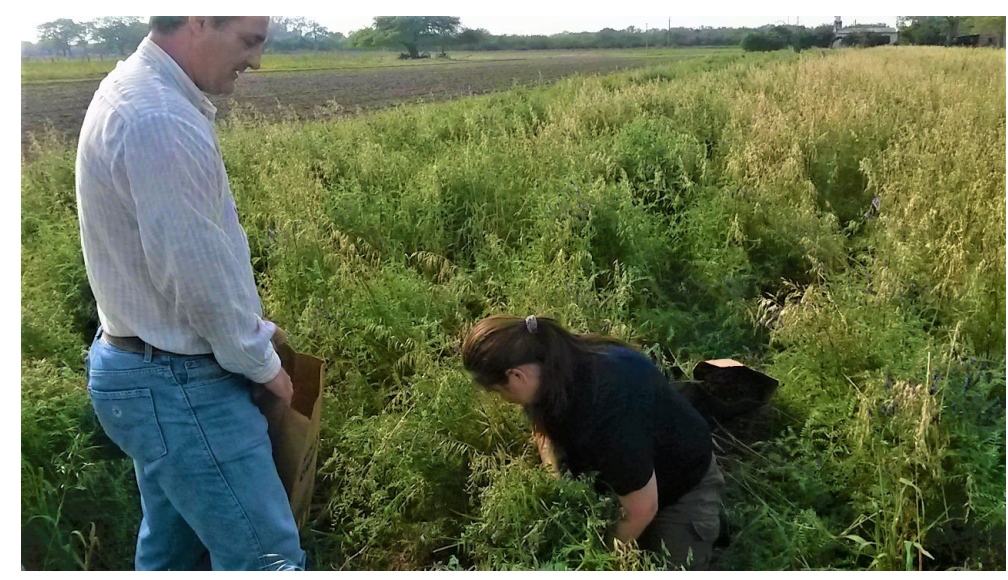

Figura 1. Muestreo de la biomasa generada por los cultivos de servicios. 
Se realizaron 3 talleres sobre esta práctica en instalaciones de productores demostrativos. Los mismos tuvieron como destinatarios técnicos, productores y público en general. En los talleres se comunicaron los resultados obtenidos a nivel de procesos y de la aplicación de la práctica (Figura 2). Se promovió el desarrollo de una conciencia crítica en cuanto al uso de los recursos naturales y sus potencialidades, que contribuyan a establecer relaciones de equidad en el marco del desarrollo sustentable. Además, al analizar los resultados se discutió, junto con los productores, acerca de los beneficios del agregado de la materia orgánica sobre la fertilidad física y biológica, versus los de los fertilizantes inorgánicos.

En la Figura 3 se puede observar el aporte de materia seca $\left(\mathrm{kg} \mathrm{Ms.ha}^{-1}\right)$ obtenida por los productores demostradores. El promedio fue de $5000 \mathrm{~kg} \mathrm{ha}^{-1}$ aproximadamente, excepto en el productor 3, el cual realizó la experiencia debajo de invernadero con suelo fertilizado y obtuvo mayor producción de biomasa.

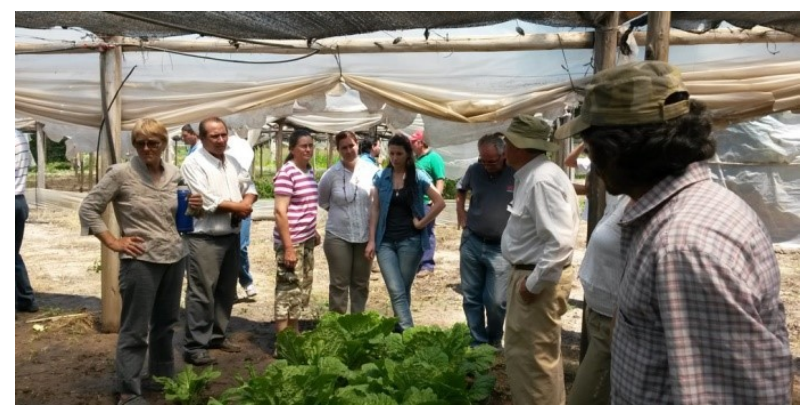

Figura 2. Taller en el campo del productor Godoy.

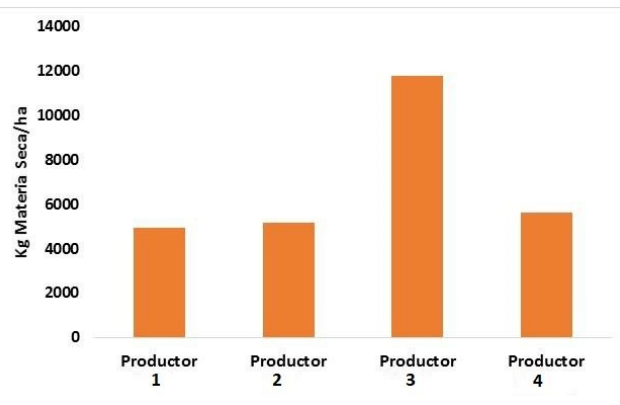

Figura 3. Producción de Materia Seca (kg de materia seca ha ${ }^{-1}$ ) del cultivo de servicio en los cuatro productores demostradores.

En los distintos talleres se realizó la entrega de boletines con información técnica sobre la práctica y los resultados zonales (Figura 4).

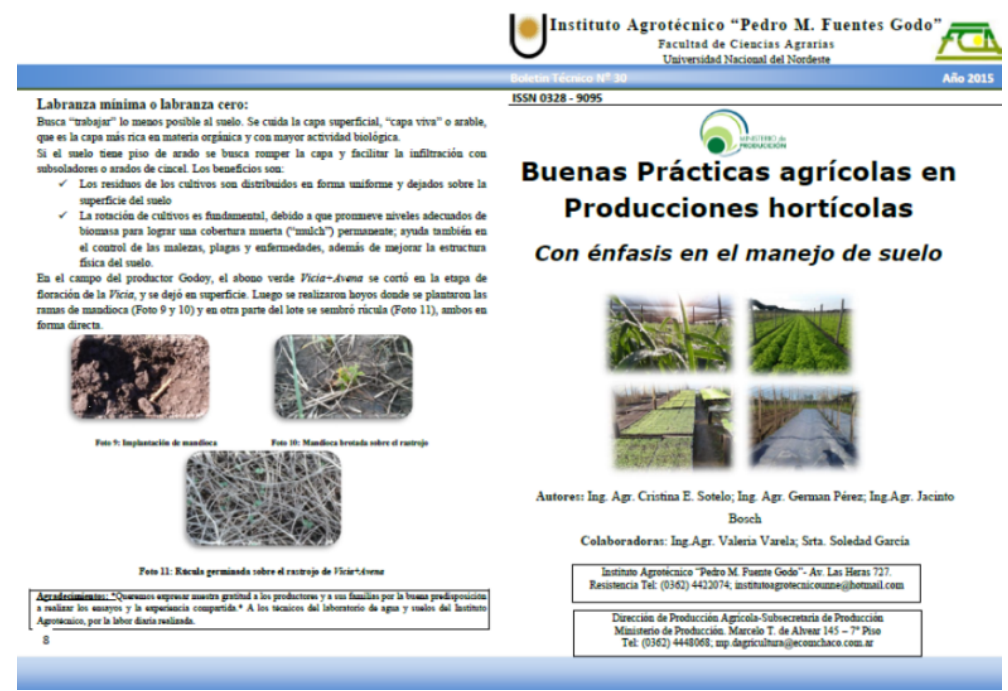

Figura 4. Boletín técnico entregado a los asistentes de los talleres.

Durante el desarrollo del proyecto los productores, estudiantes, técnicos y docentes fueron invitados a comentar los avances en el programa "La hora del Agrotécnico" de la radio de la Universidad Nacional Tecnológica (UTN).

\section{Experiencias en Compostaje}

El compost es un producto que se obtiene durante el proceso llamado compostaje. Dicho proceso transforma sustratos orgánicos heterogéneos y frescos en materia orgánica estabilizada, dióxido de carbono, agua y minerales. Es un proceso biooxidativo controlado, llevado adelante por numerosos microorganismos, que implica el paso por una etapa termofílica con liberación temporaria de fitotoxinas y una etapa de maduración. Como con- 
Carnicer, S. et al.: Prácticas sustentables de mejoramiento de suelo.

secuencia de las elevadas temperaturas que se alcanzan durante este proceso, se eliminan semillas de malezas y microorganismos patógenos que puedan presentarse con el residuo (Costa et al., 1991; Lavado, 2012; Sánchez de Pinto et al., 2013).

Se trabajó con productores de la zona periférica del Gran Resistencia junto a personal de la Secretaría de Agricultura Familiar de la Nación (SAF) y con técnicos de la EEA del INTA de Colonia Benítez.

Con la SAF se trabajó en la zona de Puerto Vilelas y productores orgánicos de Puerto Tirol en el establecimiento "Tierra sin mal".

Con personal del INTA se trabajó con la huerta de un predio de la organización sin fines de lucro Rehabilitación de Marginados (REMAR) ubicado en Resistencia y con el personal del Museo Casa Jardín Botánico Augusto Schulz (Colonia Benítez), lugar de encuentro para muchos pequeños productores donde comparten experiencias y jornadas.

En los lugares mencionados se realizaron pilas utilizando residuos de cada establecimiento o aledaños. Además, se suministraron los elementos necesarios para realizar adecuadamente las actividades como ser, termómetros, carretillas, palas de punta y palas anchas para el proceso de compostaje.

El proceso de compostaje, como ya se mencionó, fue realizado participativamente en cada establecimiento, y se realizó de la siguiente manera:

- Relevamiento de la materia prima en cada establecimiento o sitio.

- Acopio de dichos materiales.

- Reducción del tamaño de los residuos en los casos necesarios.

- Armado de la pila: consistió en mezclar de manera correcta los materiales y adecuar el contenido de humedad.

- Se realizó el seguimiento de la temperatura periódicamente por medio del termómetro y planilla entregado a cada establecimiento.

- Se realizaron los volteos y corrección de humedad pertinentes cada vez que el proceso lo requirió (Cuando las temperaturas de las pilas caían debajo de $55^{\circ} \mathrm{C}$ )

- Una vez que las pilas alcanzaron temperatura correspondiente a etapa termofílica se realizaron análisis de germinación de semillas en el predio de los productores como indicador de fitotoxicidad (indicador de madurez). Además se realizaron algunas determinaciones químicas en el laboratorio del Instituto Agrotécnico.

Luego de finalizado el proceso de compostaje se realizó un taller (Figura 5) en las instalaciones del Museo Casa Jardín Botánico Augusto Schulz para comentar las tareas que se realizaron y para mostrar el efecto de este abono sobre las propiedades del suelo. Se promovió la valorización de los residuos, la elaboración de compost y los beneficios al suelo y a las plantas. También se realizaron demostraciones prácticas con valija de campaña sobre los beneficios que brinda el compost al incorporarlos al suelo. En la misma se realizó la entrega de folletos con información técnica sobre la práctica y sus beneficios (Figura 6).

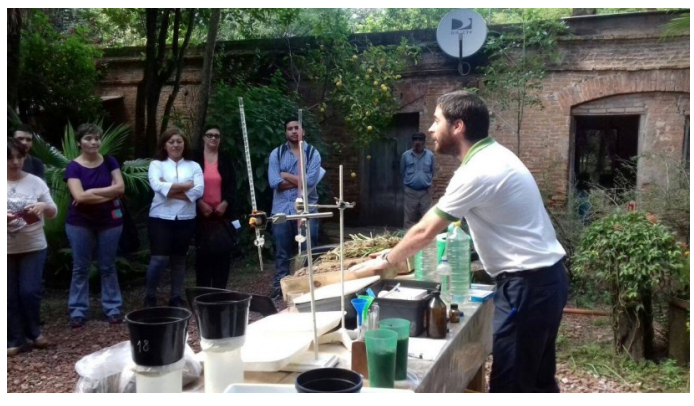

Figura 5. Taller sobre elaboración de compost y demostración de la valija de campaña.

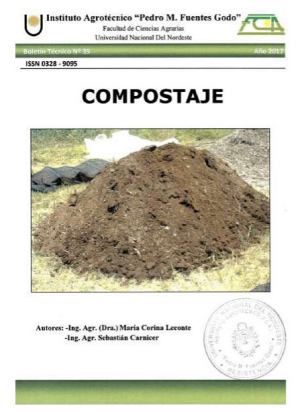

Figura 6. Boletín de compostaje $\mathrm{N}^{\circ} 35$. 


\section{Talleres de suelo}

Dentro de los proyectos, también se realizaron cinco talleres a campo en distintas zonas de la provincia de Chaco invitando a productores, alumnos de distintas carreras agropecuarias, docentes de escuelas rurales y público interesado.

Las actividades desarrolladas en los mismos estuvieron basadas en la "Guía Elemental Didáctica para la enseñanza de la conservación del agua y del suelo en el aula y exterior” (Mac Donald, 1973). A los asistentes se les demostró mediante distintos experimentos el efecto del agua sobre un suelo con y sin cobertura, se evidenció el efecto agregante de la materia orgánica en porciones de suelo, se mostró la cantidad de suelo que arrastra un suelo con poca materia orgánica versus uno con buen contenido y alta infiltración (Figura 7).

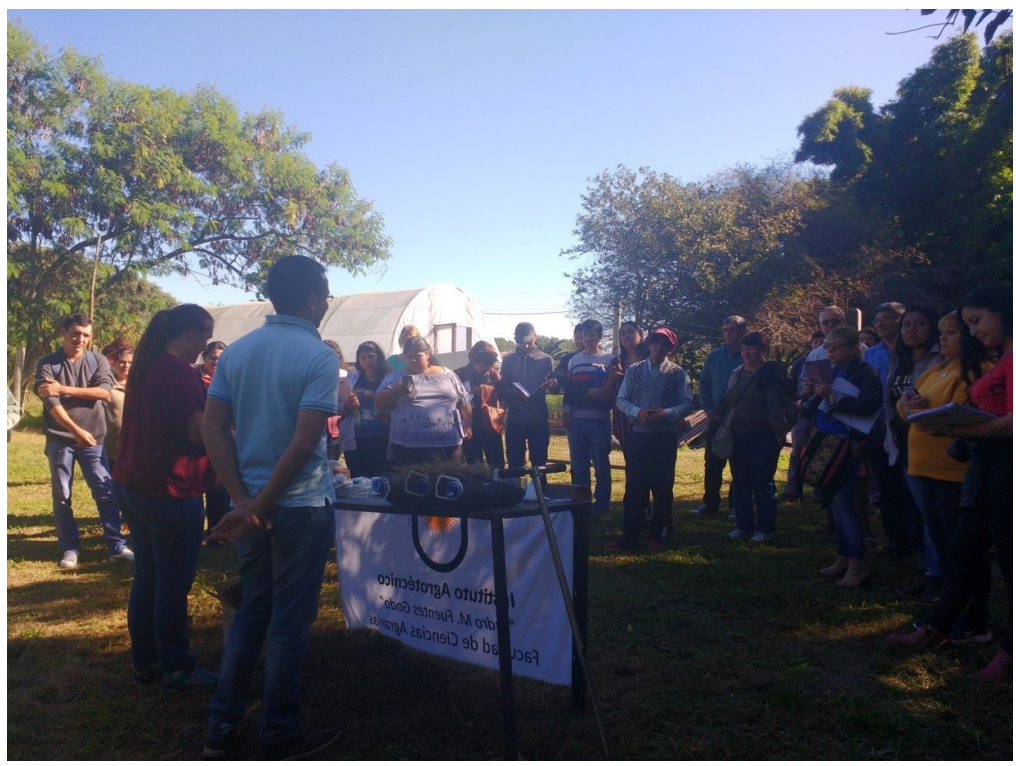

Figura 7. Taller de suelo y demostración del uso de la valija de campaña.

Por otra parte se demostró el uso de la valija de campaña la cual posee reactivos para determinar $\mathrm{pH}$, nitratos, nitritos, amonio, fósforo, materia orgánica y otros elementos.

Finalmente se comentó sobre algunos aspectos básicos del muestreo de suelo y cómo transportar la muestra al laboratorio de análisis. En relación a esto, se está confeccionando un boletín técnico de muestreo de suelos para entregar a los productores.

En cuanto a las actividades realizadas con los alumnos, las mismas consistieron en tareas de gabinete, laboratorio y de campo. Es importante destacar que ellos tienen una doble función en estos trabajos, siendo emisores y receptores de los conocimientos planteados. Por tal motivo, sus tareas se realizaron con el acompañamiento de los profesionales responsables de cada área y con los directores, co-directores y coordinadores de los proyectos. Las mismas fueron:

Reunión informativa inicial entre docentes y alumnos sobre los motivos, fundamentos técnicos y objetivos planteados en cada proyecto.

Tareas de campo: Consistieron en salidas a campo, prácticas demostrativas y talleres.

Tareas de laboratorio: realizadas con los responsables de los laboratorios.

Tareas de gabinete: Sistematización e interpretación de datos de las prácticas o ensayos de campo.

Participación en talleres como personal de apoyo y logística.

Los alumnos que participaron activamente durante los años de estos proyectos fueron quince. Dos de ellos realizaron su trabajo final de graduación de la carrera de Ingeniería Agronómica y tres realizaron adscripciones con los temas abordados. 
Carnicer, S. et al.: Prácticas sustentables de mejoramiento de suelo.

\section{CONSIDERACIONES FINALES}

Se logró un trabajo en equipo entre los distintos actores de los proyectos estudiantes, productores y profesionales de las distintas instituciones involucradas.

Los alumnos tuvieron la oportunidad de aplicar conocimientos teóricos adquiridos durante sus años de estudio al llevarlos a la práctica en tareas como muestreo de suelo, análisis físicos, químicos y biológicos de suelo y compost. También conocieron la realidad que afronta el productor en su vida laboral cotidiana y cómo interactuar en los procesos de generación del conocimiento en la educación no formal.

En cuanto a los productores, ellos mostraron predisposición e interés por las prácticas recomendadas. En los años en los que se han realizado los proyectos han sido cerca nueve los productores llamados "Demostradores", los cuales fueron los beneficiarios directos de los proyectos, mientras que cerca de 100 personas participaron de los talleres ofrecidos. A su vez, se logró desarrollar una conciencia crítica sobre ciertas prácticas agrícolas realizadas, viéndolos comprometidos hacia un cambio de paradigma. También se afianzó el vínculo laboral y de respeto mutuo entre ellos y los profesionales de las distintas instituciones participantes que trascendió a los proyectos específicos en los que estaban involucrados cada uno, logrando un espacio coparticipativo y dinámico.

Para a la difusión de los resultados de los ensayos se confeccionaron folletos y boletines técnicos sobre las temáticas de abonos verdes y compostaje que fueron distribuidos entre los asistentes a los talleres y actualmente están disponibles online.

\section{BIBLIOGRAFÍA}

Bejarano, C. (2011). Los actores de la Extensión Universitaria. Un Saber Hacer para la construcción de un enfoque CTS. XI Congresp Iberoamericano de Extensión Universitaria. Santa Fe. Argentina.

Castro-Rincón, E., Mojica-Rodríguez, J.E., Carulla-Fornaguera, J.E. y Lascano-Aguilar, C.E. (2018). Evaluación de leguminosas como abono verde en cultivos forrajeros para ganaderías en el Caribe seco colombiano. Agronomía Mesoamericana, 29 (3): 597-617. https://dx.doi.org/10.15517/ ma.v29i3.32350

Chilon Camacho, E. (2018). El Paradigma "Suelo Vivo". Revista de la Carrera de Ingeniería Agronómica UMSA. Apthapi 4 (2):1148-1172. http://ojs.agro.umsa.bo/index.php/ATP/article/view/250/245

Costa, F., García, C., Hernández, T. y Polo, A. (1991). Residuos orgánicos urbanos. Manejo y utilización. Consejo Superior de Investigaciones Científicas (CSIC)-CEBAS, Murcia, España. 181 p.

Cuellas, M., Delprino, M.R., D'Angelcola, M.E., Valenzuela, O., Czepulis, J., Del Pardo, K., Ciaponi, M. y Mitidieri, M. (2018). Evaluacion de la calidad de los suelos horticolas periurbanos mediante el uso de indicadores. Boletín "Manejo de los suelos hortiflorícolas" Modelo agua y sustrato (PNHFA 1106082). pp 9-12.

Damian Suclupe, M.J., Gonzáles Veintimilla, F., Quiñones Paredes, P. y Terán Iparraguirre, J. R. (2018). Plan de enmiendas, yeso agrícola, compost mejorado y enriquecido con EM y humus de lombriz, para mejorar el suelo. Arnaldoa 25 (1): 141-158. http://doi.org/10.22497/arnaldoa.251.25109

Deagustini, C.A., Domínguez, G.F., Agostini, M.A., Studdert, G.A., Tourn, S.N. (2017). Vicia como cultivo puente y sistemas de labranza: efecto sobre propiedades físicas del suelo. Ciencia del Suelo (Argentina) 35(2): 325-335.

Lavado, R.S. (2012). Origen del compost, proceso de compostaje y su potencialidad de uso. En Mazzarino, M. J y Satti, P. (Eds.) Compostaje en la Argentina: Experiencias de producción, calidad y uso. 1ra ed., Buenos Aires, Editora Orientación Grafica. pp 3 - 12.

Mac Donald, E.A. (1973). Guía elemental didáctica para la enseñanza de conservación del agua y del suelo en el aula y exterior. Instituto Agrotécnico, Universidad Nacional del Nordeste; Ministerio General de Educación; Instituto Nacional de Tecnología Agropecuaria.

Marca-Huamancha, C., Borjas-Ventura, R., Rebaza-Fernández, D., Bello-Amez, S., y Julca-Otiniano, A. (2018). Efecto de la fertilización mineral y de un fertilizante biológico en piña $[<i>$ Ananas comosus $<1$ i\&gt; (L.) Merr.] en el cultivar MD2 ('Golden'). Revista Colombiana De Ciencias Hortícolas, 12 (1): 59-68. https://doi.org/10.17584/rcch.2018v12i1.7901

Martín, G.M. y Rivera, R. (2004). Mineralización del nitrógeno incorporado con los abonos verdes y su participación en la nutrición de cultivos de importancia económica. Cultivos Tropicales. 25(3): 89-96. 
Martín, S., Bertsch, F. y Castro, L. (2017). Efecto del manejo orgánico y convencional sobre propiedades bioquímicas de un andisol y el cultivo de papa en invernadero. Agronomía Costarricense 41(2): 27-46.

Orozco Corral, A. L., Valverde Flores, M. I., Martínez Téllez, R., Chávez Bustillos, C. y Benavides Hernández R. (2016). Propiedades físicas, químicas y biológicas de un suelo con manzano biofertilizado. Terra Latinoamericana 34: 441-456.

Pazos-Rojas, L.A., Marín-Cevada, V., Morales García, Y.E., Baez, A., Villalobos-López, M.A., PérezSantos, M. y Muñoz-Rojas, J. (2016). Uso de microorganismos benéficos para reducir los daños causados por la revolución verde. Revista Iberoamericana de Ciencias. Vol 3 (7): 72-85. http:// www.reibci.org/publicados/2016/dic/2000114.pdf

Resolución Conjunta (5/2018). Secretaría de regulación y gestión sanitaria y secretaría de alimentos y bioeconomía. Fecha de publicación en el Boletín Oficial 21/11/2018.

Sánchez de Pinto, M.I., Rodriguez, G.V., Ferreyra Grassi, M.F., Umbides, R. y Polo, A. (2013). Cambios físico-químicos y biológicos durante el compostaje de residuos biodegradables de un feedlot vacuno. En: Albanesi, Ada (Ed). Microbiología Agrícola: un aporte de la Investigación en Argentina. $2^{\mathrm{a}}$ ed. Tucumán. Magma Publicaciones. 500 pp.

Sasal, C., Andriulo, A., Ullé, J., Abrego, F.y Bueno, M. (2009). Efecto de diferentes enmiendas sobre algunas propiedades edáficas en sistemas de producción hortícola del centro norte de la región pampeana. Informe Técnico 2009 del Centro Regional Buenos Aires Norte. Edición INTA. 257pp.

Valentinuz, C., Gutierrez, O.V., Thornton, R., Tort, M.I. y Carrapizo, V. (2005). Metodología de enseñanza-aprendizaje aplicadas a la extensión rural. Editorial INTA. 155 pp.

Weil, R.R. y Magdoff, F. (2004). 1. Significance of Soil Organic Matter to Soil Quality and Health. En: Magdoff, F y Weil, R.R. (Eds.). Soil Organic Matter in Sustainable Agriculture. 43 pp. 